\title{
MODELO MATEMÁTICO PARA CORTES DE BARRAS DE AÇO NO PROCESSO DE FABRICAÇÃO DE TRIÂNGULOS
}

\author{
H. C. WAVRZYNCZAK, G. ULBRICHT* e E. S. M. TEIXEIRA \\ Instituto Federal de Educação, Ciência e Tecnologia de Santa Catarina \\ gerson.ulbricht@ifsc.edu.br*
}

Artigo submetido em outubro/2015 e aceito em dezembro/2015

DOI: $10.15628 /$ holos.2015.3605

\section{RESUMO}

Uma das preocupações das empresas em seus processos fabris é quanto a melhor utilização da matéria-prima, trazendo como consequência a redução ou elevação no custo de fabricação. Sendo assim, no desenvolvimento deste trabalho é apresentado um modelo matemático de otimização com objetivo de minimizar o desperdício de matéria-prima com o uso de ferramentas da pesquisa operacional. Trata-se assim, de um problema de corte unidimensional de barras de aço cilíndricas para a fabricação de triângulos. Em um primeiro estágio foi estabelecido o maior número possível de padrões de corte e em um segundo estágio, foram definidos quais padrões seriam utilizados em quantidade para atender às demandas previstas. Para validação do modelo, este foi aplicado em uma empresa fabricante de instrumentos musicais, no processo de fabricação de triângulos de quatro medidas onde foi trabalhado com corte de barras, buscando reduzir ao máximo os desperdícios. Assim, obteve-se uma perda teórica de $0,3 \%$ do total de barras de aço, menos do que a empresa atinge atualmente. Os testes realizados tem mostrado boas perspectivas quanto à redução significativa nos desperdícios o que demonstra que o modelo se apresenta como um boa ferramenta no auxílio à decisão da gestão de recursos.

PALAVRAS-CHAVE: triângulos, cortes unidimensionais, minimização de perdas, modelo matemático

\section{MATHEMATICAL MODEL FOR STEEL BARS CUTS IN THE PROCESS OF MANUFACTURING OF TRIANGLES}

\begin{abstract}
One of the concerns of the companies in their manufacturing processes is about the better use of the raw material, bringing the effect of reducing or increase the manufacturing cost. Thus, the development of this paper presents a mathematical model of optimization in order to minimize the waste of raw material with the use of operations research tools. It is thus a one-dimensional cutting problem of cylindrical steel bars for the manufacture of triangles. In a first stage it is set the largest possible number of cutting patterns, and in a second stage, defined patterns which would be used in
\end{abstract}

an amount to meet anticipated demands. To validate the model, this was applied to a manufacturer of musical instruments in the manufacturing process of four measures of triangles where was worked with cutter bars, trying to reduce to the minimum waste. Thus, there was obtained a theoretical loss of $0.3 \%$ of the total steel bars, less than the company currently reach. The tests have shown good prospects as a significant reduction in waste which shows that the model is presented as a good tool to aid the decision of resource management. 


\section{INTRODUÇÃO}

O aumento da competitividade entre as empresas faz com que estas busquem melhorar seu rendimento por meio da utilização correta dos recursos disponíveis. Estas melhorias consistem em procurar estratégias que contribuam para o melhor aproveitamento da matéria-prima, dos recursos humanos e tecnológicos e demais fatores que possam vir a colaborar para a eficácia do processo produtivo.

Ferramentas matemáticas tem sido amplamente difundidas nos últimos anos, e sua utilização no ambiente industrial, contribui para melhorias no processo de produção de modo a evitar gastos excessivos de recursos e desperdícios de produção.

Verificando a forma como ocorre o processo de cortes de barras em uma empresa fabricante de instrumentos musicais percebeu-se um significativo desperdício de material. Estas perdas associadas à fabricação de triângulos são significativas pela demanda e representatividade deste produto para a empresa analisada. Viu-se então que ferramentas matemáticas poderiam agregar valor ao processo ao propiciar um estudo teórico de implantação de um modelo capaz de contribuir para a redução dessas perdas. Assim, baseado nas ideias relacionadas aos problemas de corte unidimensionais verificou-se a possibilidade de implantar um modelo de otimização nesta empresa, onde, no processo de produção, há a necessidade de programar cortes em barras de aço com $6000 \mathrm{~mm}$ de comprimento de modo a atender à demanda para a produção de triângulos, os quais consistem em peças para instrumentos musicais com diferentes medidas.

Sendo assim, depois de verificado como se dá o processo de produção nesta empresa, onde ocorrem constantes perdas de matéria-prima de modo que não é possível seu reaproveitamento, foi formulado um modelo matemático voltado a reduzir tais desperdícios por meio do estabelecimento de um correto planejamento no processo de cortes de barras de aço. Assim, em um primeiro momento foi identificado o maior número possível de padrões de cortes para essas barras, nas medidas a serem utilizadas. Após esse procedimento, partiu-se para uma segunda etapa, de modo a elaborar e solucionar um modelo matemático que permite estabelecer quais destes padrões serão utilizados e em que quantidade, de forma a cumprir as demandas e reduzir desperdícios.

Deste modo, o objetivo deste artigo é demonstrar a utilização de ferramentas matemáticas para a otimização do processo de cortes de barras unidimensionais de uma indústria de instrumentos musicais no processo de fabricação de triângulos. Nota-se que o modelo de otimização proposto é uma importante ferramenta no apoio à decisão, contribuindo assim para o melhor aproveitamento dos recursos disponíveis na empresa.

\section{REFERENCIAL TEÓRICO}

Uma importante ciência voltada para soluções de problemas através de modelos matemáticos é a Pesquisa Operacional (PO). A ideia principal é estabelecer o melhor plano para utilização dos recursos disponíveis, os quais na maioria das vezes são limitados. A PO surgiu durante a segunda guerra mundial, onde equipes de cientistas motivados pelo objetivo de criar estratégias na tentativa de solucionar problemas de operações militares criaram os primeiros modelos de programação matemática. Devido ao desempenho dessa técnica, a mesma passou a 
ser aplicada por acadêmicos e empresários em problemas administrativos, pois trata-se de um método que facilita muito o processo de decisão (ANDRADE,2000; SILVA et al., 1998).

Arenales et al. (2007), definem Pesquisa Operacional, como uma ciência que procura elaborar métodos científicos, com finalidade de dar suporte na tomada de decisões para solucionar determinados problemas. Também está relacionado a princípios e métodos de modelagem de problemas de decisão através de técnicas e modelos matemáticos. A PO é utilizada nas mais diversas áreas, como por exemplo nas áreas de produção e logística, na prestação de serviços, na elaboração de planos de transporte, na área agrícola, entre outros (CAIXETA-FILHO, 2001; GOLDBARG e LUNA, 2005). Uma importante área de aplicação da PO são os problemas de cortes, nos quais o objetivo principal consiste em estabelecer planos de corte, sejam unidimensionais, como por exemplo cortes de barras ou bobinas, bidimensionais como cortes de chapas, ou ainda tridimensionais, de modo a atender às demandas estabelecidas pela indústria com o menor desperdício possível de matéria-prima, o que traz como consequência direta, a redução nos custos de produção, bem como contribuições relacionadas às questões ambientais (ARENALES et al., 2007).

Uma importante ferramenta matemática para solucionar problemas da PO, é a Programação Linear, a qual consiste em um processo que envolve inversões sucessivas de matrizes, sendo assim uma técnica possível de ser computacionalmente programada (SILVA et al., 1998). Para que seja resolvido um problema de programação linear, este deve ser modelado definindo qual o objetivo do problema, se é maximizar lucros, ou minimizar custos ou desperdícios por exemplo (ANDRADE,1998). Silva et al. (1998), propõem seguir um caminho em três elementos básicos para a construção de modelos matemáticos com objetivo de ordenar o raciocínio. 0 primeiro elemento é relacionado com as variáveis de decisão onde devem ser apresentadas as possíveis decisões a serem tomadas. O segundo é qual o objetivo do problema onde são relatados e identificados os porquês da tomada de decisão. Nesse elemento o objetivo sempre será voltado para calcular o valor (lucro, custo, receita, perda, etc), e o terceiro elemento são as restrições, as quais caracterizam as limitações do problema.

Para Andrade (1998) tomar uma decisão com alta grandeza de qualidade não é uma tarefa tão fácil quanto parece. Muitas vezes, decisões aparentemente inapropriadas para algumas pessoas hoje, podem-se tornar decisões bem sucedidas no futuro. Assim pode-se afirmar que uma decisão com alta qualidade é quando as mesmas asseguram a realização dos propósitos predeterminados de modo a atender sua real necessidade.

Trabalhos recentes na área da programação de cortes têm sido aplicados no ambiente industrial. Uma importante aplicação desta classe de problemas é encontrada em Loeblein et al. (2012). Neste trabalho os autores propõem um modelo voltado ao corte de tubos onde foram descritos 28 padrões de cortes distintos, onde o modelo matemático foi implementado com a utilização de planilhas usando a ferramenta Solver. Os resultados foram aplicados em uma empresa fabricante de máquinas agrícolas e mostrou a viabilidade da implantação da pesquisa operacional na empresa. Silveira et al. (2013), destacaram em seu trabalho a aplicação da Pesquisa Operacional em uma indústria metalmecânica com objetivo de otimizar uma operação de corte de barras de aço, onde foram criadas 137 combinações de corte. A ferramenta Solver do programa Microsoft ${ }^{\odot}$ Office Excel 2007 foi utilizada para a resolução dos modelos e com isso atender a demanda necessária e minimizar o desperdício de materiais. 
Outro trabalho que pode ser citado é o de Silveira Júnior, Pinheiro e Thomaz (2004) que realizaram um estudo em uma indústria metalmecânica direcionado a um problema de corte de guilhotinados para bobinas de aço, onde apresentaram modelos matemáticos afim de minimizar perdas, os quais foram resolvidos com auxílio do software LINDO. Já Oliveira, Salvador e Silva (2013), realizaram um estudo em uma indústria papeleira, com o objetivo de minimizar a quantidade de resíduos gerados pelo processo de corte de bobinas de papel, onde encontraram uma solução ótima para o problema.

Os problemas de cortes unidimensionais podem ser aplicados em diversas áreas industriais, como por exemplo, no corte de barras e de bobinas. Buscando reduzir os desperdícios no processo de corte de barras, foi abordado um problema prático, o qual é apresentado no capítulo a seguir.

\section{PROCESSO DE CORTES DE BARRAS PARA TRIÂNGULOS}

Buscando implementar o problema de cortes em uma caso prático, foi realizado um estudo de caso numa empresa fabricante de instrumentos musicais. Assim identificou-se o processo de fabricação de triângulos com potencial para o estudo, pois o produto possui várias medidas e um mesmo tipo de material. Assim, segundo Frungillo (2003), o triângulo é um instrumento musical formado por uma barra cilíndrica dobrada em dois pontos até que uma extremidade se encontre com a outra, tomando a forma de um triângulo equilátero. Trata-se de um instrumento de percussão com o som metálico e agudo de longa duração semelhante a uma pequena bigorna. Deste modo, quando suspenso por um cordão e percutidos com um pequeno bastão, emitem o som que pode ter sua sonoridade alterada com o uso da própria mão do instrumentista.

\subsection{Características dos produtos fabricados}

A empresa selecionada para o estudo de caso é uma fabricante de instrumentos musicais situada no norte de Santa Catarina. Entre os vários produtos que fabrica, o triângulo é um dos mais vendidos, sendo que o processo de fabricação é composto de basicamente duas etapas, sendo corte e dobra. O material utilizado para todos os modelos de triângulos fabricados pela empresa são barras de aço cilíndricas de $8 \mathrm{~mm}$ de diâmetro por $6000 \mathrm{~mm}$ de comprimento. A linha de produção avaliada compreende a fabricação de quatro tamanhos de triângulos conforme mostrado na Figura 1.

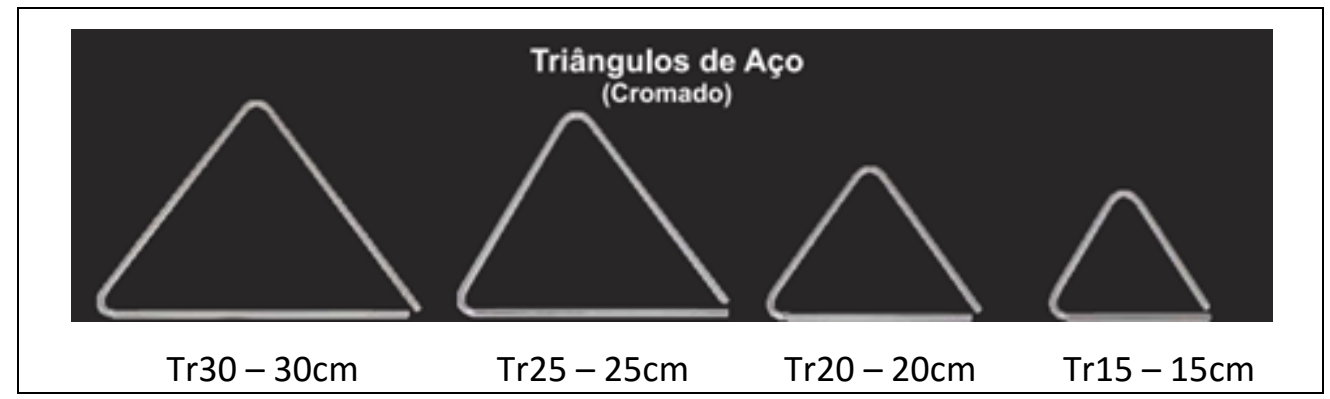

Figura 1: Tamanhos de triângulos produzidos.

Fonte: Os autores. 
Cada um dos modelos de triângulos produzidos possui uma medida lateral aproximada ao tamanho nominal do triângulo, porém devido à suas dobras e a aproximação das extremidades, a medida linear possui um tamanho específico para cada triângulo. Assim, o comprimento linear de barra de aço convenientemente cortado e dobrado forma uma nova dimensão. Esta deve ser utilizada como referência para o corte das barras de aço de cada triângulo, conforme a Tabela 1.

Tabela 1: Medidas a serem cortadas Fonte: Os autores.

\begin{tabular}{cc}
\hline Modelo & Comprimento total $(\mathbf{m m})$ \\
\hline $\operatorname{Tr} 30$ & 863 \\
$\operatorname{Tr} 25$ & 705 \\
$\operatorname{Tr} 20$ & 555 \\
$\operatorname{Tr} 15$ & 402 \\
\hline
\end{tabular}

De modo a atender as demandas estabelecidas, verificou-se que ocorriam muitas perdas de matéria-prima neste processo de fabricação da empresa avaliada. Sendo assim, foi verificada a necessidade de se estabelecer um modelo matemático que viesse a contribuir para redução destas perdas, justificando a aplicação do estudo de caso.

Na compreensão do processo de fabricação do produto verificou-se que o desperdício de matéria-prima é formado basicamente por dois tipos de perdas, a primeira delas pela não utilização de pedaços de barras que não alcançam as medidas desejadas, e o segundo tipo de perda ocasionada pela largura da serra que proporciona os cortes. Definiu-se também que não haveria alteração no processo de fabricação da empresa, seguindo-se exatamente as etapas de fabricação atuais. A Figura 2 mostra os dois tipos de perdas no processo de cortes de barras para triângulos.

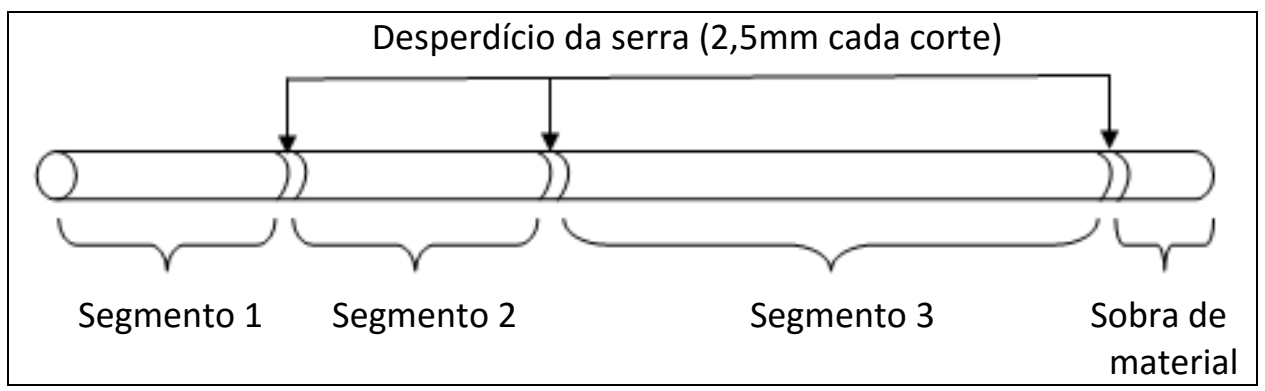

Figura 2: Tipos de desperdício no processo de cortes. Fonte: Os autores.

A forma de cortar uma barra é chamada de padrão de corte. Assim, no tópico a seguir são apresentados os padrões de cortes a serem considerados para a resolução do problema.

\subsection{Padrões de corte}

O processo de corte dos quatro tipos de triângulos ocorre por meio de uma serra, gerando desperdícios de material. A perda total ocasionada pela serra durante o processo de corte varia 
em função da quantidade de segmentos cortados em cada barra de aço de $6000 \mathrm{~mm}$, onde cada corte ocasiona uma perda de $2,5 \mathrm{~mm}$ de barra.

Considerando as diferentes medidas a serem cortadas, bem como o desperdício de material em cada corte, com o auxílio de uma planilha eletrônica foram identificados 160 diferentes padrões de corte.

$\mathrm{Na}$ Tabela 2 são apresentados alguns exemplos dos padrões encontrados bem como os respectivos desperdícios.

Tabela 2: Exemplos de padrões de cortes

Fonte: Os autores.

\begin{tabular}{c|ccccccccc}
\hline Medida do segmento & $\mathbf{P}_{\mathbf{1}}$ & $\mathbf{P}_{\mathbf{2}}$ & $\mathbf{P}_{\mathbf{3}}$ & $\mathbf{P}_{\mathbf{4}}$ & $\mathbf{P}_{\mathbf{5}}$ & $\ldots$ & $\mathbf{P}_{158}$ & $\mathbf{P}_{159}$ & $\mathbf{P}_{160}$ \\
\hline $863 \mathrm{~mm}$ & 6 & 6 & 6 & 5 & 5 & $\ldots$ & 0 & 0 & 0 \\
$705 \mathrm{~mm}$ & 1 & 0 & 0 & 2 & 1 & $\ldots$ & 0 & 0 & 0 \\
$555 \mathrm{~mm}$ & 0 & 0 & 1 & 0 & 1 & $\ldots$ & 2 & 1 & 0 \\
$402 \mathrm{~mm}$ & 0 & 1 & 0 & 0 & 1 & $\ldots$ & 12 & 13 & 14 \\
Total de segmentos $(\mathrm{mm})$ & 5883 & 5580 & 5733 & 5725 & 5977 & $\ldots$ & 5934 & 5781 & 5628 \\
Consumo da serra $(\mathrm{mm})$ & 17,5 & 17,5 & 17,5 & 17,5 & 20 & $\ldots$ & 35 & 35 & 35 \\
Desperdício de material $(\mathrm{mm})$ & 99,5 & 402,5 & 249,5 & 257,5 & 3,0 & $\ldots$ & 31,0 & 184,0 & 337,0 \\
\hline
\end{tabular}

O modelo matemático proposto é voltado para a minimização do desperdício de material. Na Tabela 2, esse desperdício é calculado conforme a seguir.

No padrão de corte $P 1$ por exemplo, são cortados 6 segmentos de $863 \mathrm{~mm}$, mais 1 segmento de $705 \mathrm{~mm}$, o que totaliza a utilização de $5883 \mathrm{~mm}$ do total de barra de $6000 \mathrm{~mm}$ (6 x $863 \mathrm{~mm}+1 \mathrm{x}$ $705 \mathrm{~mm}=5883 \mathrm{~mm}$ ). Porém para efetuar esses cortes há o desperdício ocasionado pelo processo de corte. Neste caso, como foram obtidas 7 peças (ou segmentos), foram consequentemente realizados 7 cortes, ocasionando um desperdício ocasionado pela serra de $17,5 \mathrm{~mm}(7 \times 2,5 \mathrm{~mm}=$ $17,5 \mathrm{~mm})$. Sendo assim, o total da barra consumido no padrão de corte P1 foi de $5900,5 \mathrm{~mm}$ $(5883 \mathrm{~mm}+17,5 \mathrm{~mm}=5900,5 \mathrm{~mm})$. Como a barra que foi cortada possui originalmente $6000 \mathrm{~mm}$, há um desperdício de material de $99,5 \mathrm{~mm}(6000 \mathrm{~mm}-5900,5 \mathrm{~mm}=99,5 \mathrm{~mm})$. Nota-se que esse segmento de $99,5 \mathrm{~mm}$ não é útil para a empresa, pois a menor medida utilizada no processo de fabricação de triângulos é de $402 \mathrm{~mm}$.

Observa-se que, no padrão P2 há um desperdício de material de 402,5mm. Sendo que a menor medida utilizada no processo de fabricação é de $402 \mathrm{~mm}$, poderia se pensar que esse não seria um desperdício, pois poderia ser, nesse caso, utilizado na empresa. Porém há de se considerar que a utilização desse segmento de $402,5 \mathrm{~mm}$ geraria mais um corte, o que pelo consumo ocasionado pela serra, de $2,5 \mathrm{~mm}$, resultaria assim numa peça de $400 \mathrm{~mm}$, a qual não teria utilidade no processo de fabricação.

Conforme já comentado, além do desperdício de material em cada padrão de corte, no modelo matemático foi considerado o desperdício ocasionado pela espessura da serra quando esta efetua o corte de cada peça. Esse tipo de desperdício sempre ocorrerá, porém pode ser reduzido, de modo que, quanto menor a quantidade de cortes em cada barra de $6000 \mathrm{~mm}$, menor será o 
desperdício ocasionado pela serra. Por exemplo, no padrão de corte $\mathrm{P}_{1}$ ocorrem 7 cortes de modo que estes somam um desperdício de $17,5 \mathrm{~mm}$ de perdas $(7 \times 2,5 \mathrm{~mm}=17,5 \mathrm{~mm})$. Já no padrão de corte $\mathrm{P}_{160}$ ocorrem 14 cortes totalizando um desperdício de $35 \mathrm{~mm}$ de material $(14 \times 2,5 \mathrm{~mm}=35$ $\mathrm{mm})$, somente ocasionado pela serra.

Deste modo, no tópico a seguir apresenta-se o modelo matemático de programação linear para o problema apresentado.

\subsection{Modelo matemático}

Para a formulação do modelo matemático foram estabelecidas demandas para cada um dos modelos de triângulo, conforme dados fornecidos pela empresa. Assim, na tabela 3 são apresentadas as demandas para cada um dos quatro tipos de triângulos de modo a atender os pedidos mensais de clientes.

Tabela 3: Demandas mensais para cada um dos quatro modelos de triângulos Fonte: Os autores.

\begin{tabular}{cc}
\hline Modelo & Demanda (unidades) \\
\hline $\operatorname{Tr} 30$ & 1000 \\
$\operatorname{Tr} 25$ & 1500 \\
$\operatorname{Tr} 20$ & 800 \\
$\operatorname{Tr} 15$ & 800 \\
\hline
\end{tabular}

Na formulação do modelo foi considerada a existência de estoques, prática adotada pela empresa avaliada para atender as flutuações de mercado e os pedidos extras. Deste modo, utilizouse um cálculo de estoque de até $10 \%$ do valor da demanda. Neste caso, a demanda do modelo $\mathrm{Tr}$ 30 poderá variar entre 1000 e 1100 unidades. A do modelo $\operatorname{Tr} 25$, entre 1500 e 1650 unidades e a dos modelos $\operatorname{Tr} 20$ e $\operatorname{Tr} 15$ entre 800 e 880 unidades.

A partir das análises e definições prévias, desenvolveu-se um modelo matemático para o atendimento da situação, conforme sequência abaixo.

a) Objetivo do modelo: minimizar o desperdício de matéria-prima (barras de aço).

b) Variáveis de decisão:

$P_{\mathrm{j}}$ : Quantidade a ser produzida do padrão de corte $\mathrm{j}(\mathrm{j}=1, \ldots, 160)$.

c) Modelo:

Minimizar 99,50P $1+402,50 \mathrm{P}_{2}+249,50 \mathrm{P}_{3}+257,50 \mathrm{P}_{4}+3,00 \mathrm{P}_{5}+\ldots+337,00 \mathrm{P}_{160}$

Sujeito a:

(Qtde_Min_863mm) $6 P_{1}+6 P_{2}+6 P_{3}+5 P_{4}+5 P_{5}+\ldots+0 P_{160} \geq 1000$ 


$$
\begin{aligned}
& \text { (Qtde_Máx_863mm) 6P } 1+6 \mathrm{P}_{2}+6 \mathrm{P}_{3}+5 \mathrm{P}_{4}+5 \mathrm{P}_{5}+\ldots+0 \mathrm{P}_{160} \leq 1100 \\
& \text { (Qtde_Min_705mm) } 1 \mathrm{P}_{1}+0 \mathrm{P}_{2}+0 \mathrm{P}_{3}+2 \mathrm{P}_{4}+1 \mathrm{P}_{5}+\ldots+O \mathrm{P}_{160} \geq 1500 \\
& \text { (Qtde_Máx_705mm) } 1 \mathrm{P}_{1}+0 \mathrm{P}_{2}+\mathrm{OP}_{3}+2 \mathrm{P}_{4}+1 \mathrm{P}_{5}+\ldots+0 \mathrm{P}_{160} \leq 1650 \\
& \text { (Qtde_Min_555mm) } 0 P_{1}+O P_{2}+1 P_{3}+O P_{4}+1 P_{5}+\ldots+O P_{160} \geq 800 \\
& \text { (Qtde_Máx_555mm) } 0 P_{1}+O P_{2}+1 P_{3}+0 P_{4}+1 P_{5}+\ldots+0 P_{160} \leq 880 \\
& \text { (Qtde_Min_402mm) } 0 P_{1}+1 P_{2}+O P_{3}+O P_{4}+1 P_{5}+\ldots+14 P_{160} \geq 800 \\
& \text { (Qtde_Máx_402mm) } 0 P_{1}+1 P_{2}+0 P_{3}+0 P_{4}+1 P_{5}+\ldots+14 P_{160} \leq 880 \\
& P_{1}, P_{2}, P_{3}, \ldots, P_{160} \geq 0
\end{aligned}
$$

\subsection{Resolução do modelo matemático}

O modelo foi resolvido utilizando-se a ferramenta Solver do programa Microsoft ${ }^{\odot}$ Office Excel, onde foram obtidas as quantidades de produtos a serem fabricadas de cada padrão de corte, conforme apresentado na Tabela 4, o que corresponde à solução ótima do problema.

Tabela 4: Quantidades a serem fabricadas de cada padrão de corte. Fonte: Os autores.

\begin{tabular}{c|cccccc}
\hline Medida & $\mathbf{P}_{\mathbf{8}}$ & $\mathbf{P}_{\mathbf{9}}$ & $\mathbf{P}_{\mathbf{2 8}}$ & $\mathbf{P}_{\mathbf{1 3 3}}$ & $\mathbf{P}_{\mathbf{1 4 9}}$ & $\mathbf{P}_{\mathbf{1 5 0}}$ \\
\hline $863 \mathrm{~mm}$ & 5 & 4 & 2 & 0 & 0 & 0 \\
$705 \mathrm{~mm}$ & 0 & 3 & 6 & 2 & 1 & 0 \\
$555 \mathrm{~mm}$ & 3 & 0 & 0 & 6 & 0 & 10 \\
$402 \mathrm{~mm}$ & 0 & 1 & 0 & 3 & 13 & 1 \\
Total de segmentos $(\mathrm{mm})$ & 5980 & 5969 & 5956 & 5946 & 5931 & 5952 \\
Consumo da serra $(\mathrm{mm})$ & 20 & 20 & 20 & 27,5 & 35 & 27,5 \\
Desperdício de material $(\mathrm{mm})$ & 0,0 & 11,0 & 24,0 & 26,5 & 34,0 & 20,5 \\
No de vezes a ser executado & 124 & 1 & 238 & 6 & 57 & 40 \\
\hline
\end{tabular}

Com a solução do modelo matemático identificou-se que o desperdício total no processo de cortes de barras de aço para triângulos foi de $8.640 \mathrm{~mm}$, o que equivale a $0,3 \%$ do total da matéria-prima. Este valor representa a soma de todos os segmentos de barra desperdiçados (124 $\times 0 \mathrm{~mm}+1 \times 11 \mathrm{~mm}+238 \times 24 \mathrm{~mm}+6 \times 26,5 \mathrm{~mm}+57 \times 34 \mathrm{~mm}+40 \times 20,5 \mathrm{~mm}=8.640 \mathrm{~mm})$.

Os valores fornecidos pela empresa mostram que o desperdício gerado sem a aplicação do modelo de programação de cortes fica situado entre $4 \%$ a $6 \%$ do total da matéria-prima, o que indica, nestes primeiros testes, resultados favoráveis no que se refere à redução de perdas no processo produtivo. Sendo assim, ainda numa visão teórica, o desperdício de material no processo de corte de barras para triângulo pode ser reduzido consideravelmente, gerando um processo de produção com menos perdas de material e mais eficaz. 


\section{CONCLUSÃO}

O trabalho desenvolvido apresenta uma importante aplicação da Pesquisa Operacional em uma empresa de modo a auxiliar na resolução de problemas, facilitando assim a tomada de decisão. Neste caso, a resolução do problema de corte unidimensional de barras para a fabricação de triângulos é demonstrada com resultados eficientes e com poucos investimentos financeiros. É importante salientar que trata-se de uma proposta que, apesar de ideal, serve basicamente de referência para o funcionamento da empresa. Assim, é possível tomar decisões de produção e estoque deste produto em função da demanda e da condição de produção.

Assim, o objetivo principal deste artigo foi apresentar um modelo de otimização de modo a minimizar o desperdício de matéria-prima, e como consequência reduzir custos produtivos, em uma empresa fabricante de instrumentos musicais. Esta sequência de desenvolvimento do modelo matemático permite identificar novas quantidades de produção de acordo com a demanda e o estoque real de cada modelo de triângulo produzido pela empresa.

Este estudo, ainda preliminar, abre espaço para o auxílio à programação da produção e pode ser referência para a avaliação em novos produtos. Como ainda está sendo avaliado e implantado na empresa analisada, os resultados reais do uso do modelo matemático ainda não são conclusivos, porém os resultados nos primeiros testes já indicam redução gradativa nos desperdícios, o que mostra que o método aplicado pode ser uma boa ferramenta de auxílio à decisão.

Como recomendação para trabalhos futuros, além da aplicação envolvendo novas demandas, um fator a ser verificado é a comparabilidade na qualidade da solução obtida em modelos que consideram ou não a existência de estoques. Além disso, outros trabalhos que associem variabilidades podem ser considerados para estudos futuros.

\section{REFERÊNCIAS}

1. ANDRADE, E. L., Introdução à Pesquisa Operacional: Métodos e Modelos Para a Análise De Decisão. 2. ed. Rio de Janeiro: LTC, 2000.

2. ARENALES, M. et al. Pesquisa Operacional. 4a reimpressão. Rio de Janeiro: Elsevier, 2007.

3. CAIXETA-FILHO, J. V. Pesquisa Operacional: Técnicas de Otimização Aplicadas a Sistemas Agroindustriais. São Paulo: Atlas, 2001.

4. FRUNGILLO, Mário D. Dicionário de percussão. Editora UNESP: Imprensa Oficial do Estado, São Paulo. 2003.

5. GOLDBARG, M. C.; LUNA, H. C. L. Otimização Combinatória e Programação Linear: Modelos e Algoritmos. 2a. ed. Rio de Janeiro: Elsevier, 2005

6. LOEBLEIN, L. C. et al. Aplicação da Programação Linear para Minimização de Perdas de Aparas em Indústria Metal Mecânica, Revista Gestão Industrial, Ponta Grossa, PR, v. 8, p.14-29, 2012.

7. OLIVEIRA, B.; SALVADOR, R.; SILVA, V. M. D. Otimização do Problema de Corte Unidimensional: Um Estudo de Caso na Indústria Papeleira. Anais: EEPA, Encontro de Engenharia de Produção Agroindustrial, 7, Campo Mourão- PR, 2013.

8. SILVA, E. M. et al. Pesquisa Operacional. 3a. ed. São Paulo: Atlas, 1998. 
9. SILVEIRA JÚNIOR, J. A.; PINHEIRO, P. R.; THOMAZ, A. C. F. Otimização das Perdas em Cortes Guilhotinados para Bobinas de Aço na Indústria Metalmecânica, Anais, SBPO: Simpósio Brasileiro de Pesquisa Operacional, 36 p. 1759 - 1770, 2004.

10. SILVEIRA, G. M. et al. Aplicação da Programação Linear para Otimização de Cortes de Eixos na Indústria Automotiva, In: Encontro Nacional de Engenharia de Produção, 33., 2013, Salvador, BA. Anais Enegep, 2013. 